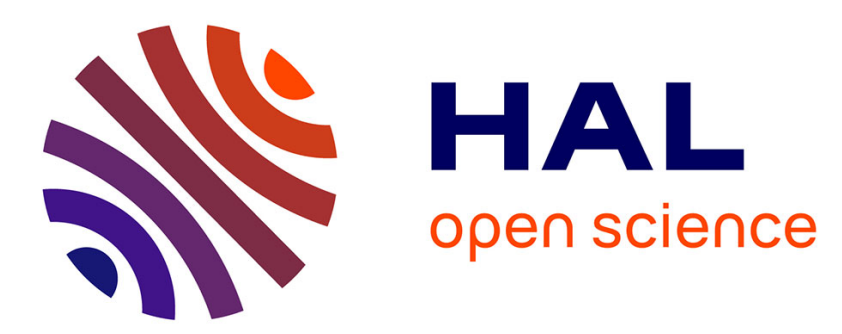

\title{
Domain duplication in ferromagnetic sandwiches
}

\author{
D Lacour, M. Hehn, O. Lenoble, A. Schuhl, C. Tiusan, K. Ounadjela
}

\section{To cite this version:}

D Lacour, M. Hehn, O. Lenoble, A. Schuhl, C. Tiusan, et al.. Domain duplication in ferromagnetic sandwiches. Journal of Applied Physics, 2001, 89 (12), pp.8006-8010. 10.1063/1.1373696 . hal02953390

\section{HAL Id: hal-02953390 \\ https://hal.science/hal-02953390}

Submitted on 30 Sep 2020

HAL is a multi-disciplinary open access archive for the deposit and dissemination of scientific research documents, whether they are published or not. The documents may come from teaching and research institutions in France or abroad, or from public or private research centers.
L'archive ouverte pluridisciplinaire HAL, est destinée au dépôt et à la diffusion de documents scientifiques de niveau recherche, publiés ou non, émanant des établissements d'enseignement et de recherche français ou étrangers, des laboratoires publics ou privés. 


\title{
Domain duplication in ferromagnetic sandwiches
}

\author{
D. Lacour, M. Hehn, ${ }^{\text {a) }}$ O. Lenoble, and A. Schuhl \\ Laboratoire de Physique des Matériaux, UMR CNRS 7556, B.P. 239, \\ 54506 Vandoeuvre lès Nancy Cedex, France \\ C. Tiusan and K. Ounadjela \\ Institut de Physique et de Chimie des Matériaux de Strasbourg, 23 rue du Loess, F-67037 \\ Strasbourg Cedex, France
}

(Received 27 September 2000; accepted for publication 29 March 2001)

\begin{abstract}
In this article, we give an overview of the domain duplication process which can occur in ferromagnetic sandwiches. A brief theoretical description of the process allows us to extract the main parameters governing the effect. It is shown that even if a domain structure exists in the hard electrode, no duplication can occur for a ferromagnetic coupling below a minimum value. Then, we address also the effects of residual domains on the nucleation field of the hard electrode to reconcile theory and experiments. (C) 2001 American Institute of Physics. [DOI: 10.1063/1.1373696]
\end{abstract}

\section{INTRODUCTION}

When two magnetic layers are separated by a 1-2-nmthick layer, as in magnetic spin valves or magnetic tunnel junctions (MTJs), a coupling between the electrodes usually takes place. Among all the possible interactions, magnetostatic interactions induced by domain walls are known to play an important role in the reversal properties of ferromagnetic electrodes in MTJs, ${ }^{1}$ and, consequently, on the tunnel magnetoresistance (TMR) signal. ${ }^{2}$ However, in those systems, the most often invoked interaction to explain ferromagnetic coupling between electrodes is the well known "orange peel" coupling originating from correlated interfaces. ${ }^{3}$ This ferromagnetic coupling associated with specific, but common, magnetic properties of the soft, and especially of the hard, magnetic layer is responsible for the duplication of the domain structure in the soft layer from the hard magnetic template. This effect has been reported earlier in the case of $\mathrm{Cu}$-based spin valves, ${ }^{4}$ recently, in the case of $\mathrm{Al}_{2} \mathrm{O}_{3}$-based tunnel junctions, ${ }^{5}$ and visualized by Kerr microscopy on those last junctions. ${ }^{6}$

When domains are duplicated, the spin valve or the tunnel junction appears to be in a fully parallel state from a giant magnetoresistance or TMR point of view, even if domains with opposing magnetization still exist in each layer. The stray field of each domain of the hard magnetic layer can either increase or decrease the effective field seen by the soft layer, depending on the orientation of the magnetization with respect to the applied field. So, this nonhomogeneous field can induce a domain structure in the soft layer. While magnetic anisotropy of the hard magnetic layer has been shown to be one key parameter, ${ }^{7}$ a simple model is proposed to review all the other parameters which play a role in the occurrence of domain duplication. It is shown here from the theory and the experiments that a minimum ferromagnetic coupling is required for duplication to occur.

\footnotetext{
${ }^{a)}$ Electronic mail: hehn@1pm.u-nancy.fr
}

\section{SAMPLE FABRICATION}

Junctions are deposited onto float-glass substrates using a sputtering system with cobalt $(\mathrm{Co})$, iron $(\mathrm{Fe})$, and aluminum (Al) targets mounted on rf, rf and dc magnetron cathodes, respectively. Details on the junction fabrication (oxidation process to make the alumina tunnel barrier, in the following denoted as $\mathrm{AlOx}$ ) and on the experimental setup used to characterize the junctions can be found elsewhere. ${ }^{5}$ The structure of the samples under study is glass $/ \mathrm{Co}(10 \mathrm{~nm}) /$ $\operatorname{AlOx}(x \mathrm{~nm}$, oxidation time $y \mathrm{~s}) / \mathrm{Co}(5 \mathrm{~nm}) / \mathrm{Fe}(20 \mathrm{~nm}) / \mathrm{Co}(5$ $\mathrm{nm}) / \mathrm{Al}(10 \mathrm{~nm})$, where the thickness of each layer is given in the brackets in nanometers. Deposition conditions of the soft $\mathrm{Co}(10 \mathrm{~nm})$ and of the hard $\mathrm{Co}(5 \mathrm{~nm}) / \mathrm{Fe}(20 \mathrm{~nm}) / \mathrm{Co}(5 \mathrm{~nm}) /$ $\operatorname{Al}(10 \mathrm{~nm})$ have been optimized such that the easy axis of both layers are parallel and that magnetization reversal in both cases occurs by nucleation and propagation of domain walls. The thickness $x$ of the aluminum layer before oxidation has been varied during this study, and in each case the oxidation time $y$ has been optimized to get the maximum TMR signal.

The geometry for current perpendicular to film plane (CPP) measurements is obtained using two different methods. The first one makes use of ex situ shadow contact masks to make cross-like junctions. A $1.5-\mathrm{cm}-$ long and $200-\mu \mathrm{m}-$ wide $\mathrm{Co}(10 \mathrm{~nm})$ electrode is first deposited onto the glass substrate. Then, a $1-\mathrm{cm}$ wide square of $\mathrm{Al}$ is deposited on top of the Co electrode, subsequently oxidized, and is covered by the $\mathrm{Co}(5 \mathrm{~nm}) / \mathrm{Fe}(20 \mathrm{~nm}) / \mathrm{Co}(5 \mathrm{~nm}) / \mathrm{Al}(10 \mathrm{~nm})$ counterelectrode, which has the same dimensions as the soft electrode. In this case, the electrodes are made with magnetic materials. The second method makes use of lithography on the asdeposited films. In this case, the complete stack is first deposited. Then, a $\mathrm{Co} / \mathrm{AlOx} / \mathrm{Co} / \mathrm{Fe} / \mathrm{Co} / \mathrm{Al}$ dumbbell-shaped electrode is first ion milled into the film to disconnect all the junctions. Then, the dumbbell pattern is etched into a disk shape down to the $\mathrm{AlOx}$ layer. So, a $\mathrm{Co} / \mathrm{AlOx} / \mathrm{Co} / \mathrm{Fe} / \mathrm{Co} / \mathrm{Al}$ disk remains on top of a Co dumbbell-shaped electrode, and electric contacts are made on the $\mathrm{Al}$ and Co layers (see, for example, Ref. 8, for more details). With those two tech- 


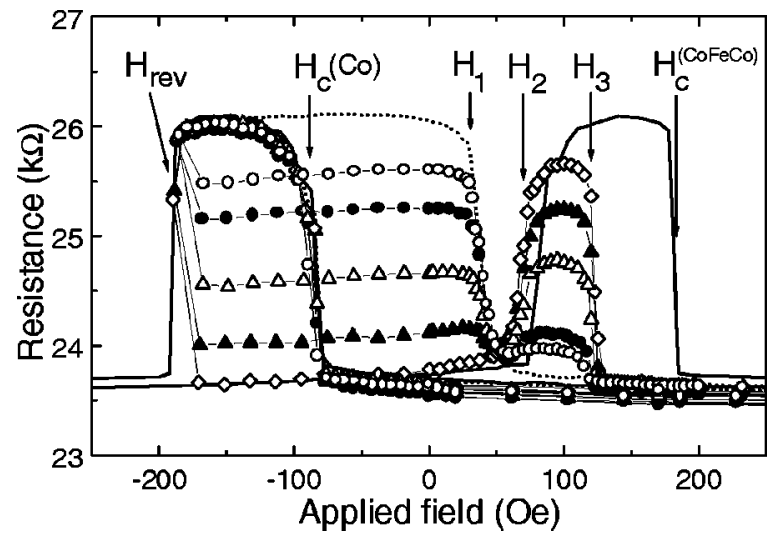

FIG. 1. Complete ( -$)$ and minor tunnel magnetoresistance loops measured on a $\mathrm{Co}(10 \mathrm{~nm}) / \operatorname{AlOx}(1.8 \mathrm{~nm}$, oxidation time $45 \mathrm{~s}) / \mathrm{Co}(5 \mathrm{~nm}) / \mathrm{Fe}(20 \mathrm{~nm}) /$ $\mathrm{Co}(5 \mathrm{~nm}) / \mathrm{Al}(10 \mathrm{~nm})$ tunnel junction made using ex situ changed masks with $200 \mu \mathrm{m}$ lateral size. The different minor cycles have been measured using different $H_{\text {rev }}$ values at which the applied field sequence is reversed. By reversing the field sequence at some negative applied field $H_{\text {rev }}$ on the minor loop, three resistance jumps with different signs appear at certain fields $H_{1}$, $\mathrm{H}_{2}$, and $\mathrm{H}_{3}$. The minor loop measured with the applied-field sequence reversed toward the positive field direction just after the $\operatorname{Co}(10 \mathrm{~nm})$ switching (dotted line) is shifted due to a bias field of -22 Oe, corresponding to a ferromagnetic coupling with the hard layer.

niques, TMR signals as high as $10 \%$ with shadow masks and $20 \%$ with lithography could be achieved.

\section{DOMAIN DUPLICATION AND TMR CYCLE}

Complete (-) and minor characteristic TMR cycles measured on a tunnel junction with $\mathrm{AlOx}(1.8 \mathrm{~nm}$, oxidation time $45 \mathrm{~s})$ made with ex situ changed shadow masks are shown in Fig. 1. In this sample, the strength of the dipolar coupling between the electrodes has been determined to be $H_{d}=22$ Oe, from the shift of the minor cycle in which only the soft layer is switched (dotted line). Then, the effective coercive field of the soft $\mathrm{Co}$ layer on the complete cycle $H_{c}(\mathrm{Co})$ is equal to $H_{c}^{\mathrm{int}}(\mathrm{Co})+H_{d}$. The first term $H_{c}^{\mathrm{int}}(\mathrm{Co})$ of the equation is the intrinsic coercive field of the Co layer, the one the layer has if it were alone, and the second term represents the dipolar coupling between the electrodes. After saturation at $850 \mathrm{Oe}$, the applied field is decreased down to -850 Oe (complete cycle) or to $H_{\text {rev }}$ (minor cycle). The resistance jumps, $\Delta R\left[H_{c}(\mathrm{Co})\right]$, occurring at the effective coercive field of the soft Co layer are equal in both cases. By reversing the step sequence of the applied field and increasing its value from -850 Oe or from $H_{\text {rev }}$ towards $850 \mathrm{Oe}$, the two cycles appear to be completely different when the applied field is again positive. In the case of complete negative saturation (complete cycle, continuous line), the cycle is symmetric and, therefore, contains two resistance jumps.

As far as the minor cycles are concerned, three resistance jumps with different signs appear at certain fields termed $H_{1}, H_{2}$, and $H_{3}$, which differ from $H_{c}(\mathrm{Co})$, $H_{c}^{\text {int }}(\mathrm{Co})$, or the effective coercive field of the hard CoFeCo layer, $H_{c}(\mathrm{CoFeCo})$. For applied fields between $H_{1}$ and $H_{2}$ in Fig. 1, the junction resistance is close to the one measured when the magnetizations of the two magnetic electrodes are in a parallel configuration. Therefore, directly across the bar-
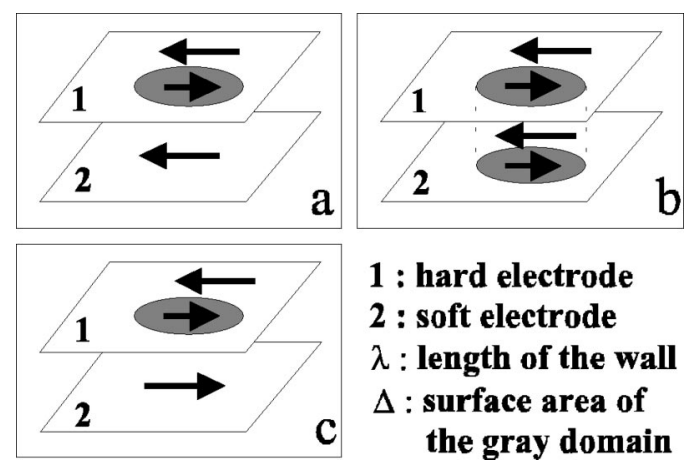

\section{1 : hard electrode \\ 2 : soft electrode \\ $\lambda$ : length of the wall \\ $\Delta$ : surface area of the gray domain}

FIG. 2. Three drawings showing the different magnetic configurations during the duplication process. In each box, sheet 1 contains the magnetic configuration of the hard layer while sheet 2 contains the one of the soft layer. The positive direction is oriented from the left to the right.

rier, the magnetizations of the two magnetic electrodes are locally parallel even if the hard magnetic layer is far from magnetic saturation. As a consequence, the domain structure of the hard layer is duplicated in the soft layer. Depending upon the choice of $H_{\text {rev }}$ around $-H_{c}(\mathrm{CoFeCo})$, the relative amount of reversed and nonreversed domains is being established in the hard layer and it changes the relative amplitude of the resistance jumps measured at $H_{1}$ and $H_{2}$. The existence of reversed domains in the hard layer creates, locally, low-resistance tunneling paths which partially short out the tunnel current and, hence, reduces the overall resistance on the $\left(H_{\text {rev }}, 0\right)$ branch.

Following the model developed earlier, ${ }^{5} H_{1}$ should be equal to $H_{c}^{\text {int }}(\mathrm{Co})-H_{d}$, while $H_{2}$ should be equal to $H_{c}^{\text {int }}(\mathrm{Co})+H_{d}$, i.e., $H_{c}(\mathrm{Co})$. This last case is not fulfilled and, sometimes, even if a domain structure exists in the hard layer, no sign of duplication of the domain structure in the soft layer can be seen in the TMR signal in a given field window of $H_{\text {rev }}$ [see, for example, curve ( $\left.-\bigcirc-\right)$ in Fig. 1]. Therefore, a more complex balance of energy must be put forward to explain the appearance of the domain structure duplication.

\section{STABILITY OF DOMAIN WALLS IN COUPLED MAGNETIC ELECTRODES}

To estimate the stability of the walls in each magnetic layer, we propose the simplified model sketched in Fig. 2. In this model, two magnetic layers are ferromagnetically coupled through a nonmagnetic layer and the magnetization of each layer is aligned with the applied field (either parallel or antiparallel). We consider that nonreversed domains exist in the hard layers, which have a total surface area $\Delta$. The domain walls have a length or perimeter $\lambda$, an energy per unit surface area $\sigma_{1}$, and no lateral extension. Let us call $t_{1}$ and $t_{2}$ the thicknesses of each magnetic layer, $M_{s 1}$ and $M_{s 2}$ the saturation magnetizations, and $J_{F}$ the interlayer coupling constant. When duplication occurs, the domains created in soft layer 2 have a total surface area $\Delta$ and the domain walls have an energy per unit surface area equal to $\sigma_{2}$.

We have to evaluate the energy of the coupled layers in three different situations: (i) with the field applied in the positive direction after negative saturation of the magnetic 
soft layer, a domain with positive magnetization remains in the magnetic hard layer, situation a with energy $\varepsilon_{a}$ [Fig. 2(a)]; (ii) with the field applied in the positive direction, a domain with positive magnetization is nucleated in the magnetic soft layer, situation b with energy $\varepsilon_{b}$ [Fig. 2(b)]; and (iii) with the field applied in the positive direction after saturation of the magnetic soft layer, a domain with negative magnetization remains in the magnetic hard layer, situation $\mathbf{c}$ with energy $\varepsilon_{c}$ [Fig. 2(c)]:

$$
\begin{aligned}
\varepsilon_{a}(H)= & -M_{s 1} H\left[2 t_{1} \Delta-S\left(t_{1}+\alpha t_{2}\right)\right] \\
& +\sigma_{1} \lambda t_{1}-J_{F}(S-2 \Delta), \\
\varepsilon_{b}(H)= & -M_{s 1} H\left(t_{1}+\alpha t_{2}\right)(2 \Delta-S) \\
& +\sigma_{1} \lambda t_{1}+\sigma_{2} \lambda t_{2}-J_{F} S, \\
\varepsilon_{c}(H)= & -M_{s 1} H\left[2 t_{1} \Delta-S\left(t_{1}-\alpha t_{2}\right)\right] \\
& +\sigma_{1} \lambda t_{1}+J_{F}(S-2 \Delta),
\end{aligned}
$$

where $\alpha$ is equal to $M_{s 2} / M_{s 1}$ and the junction surface area is equal to $S$.

We suppose that the system goes from situation $\mathbf{a}$ to $\mathbf{b}$ as soon as $\varepsilon_{a}>\varepsilon_{b}$ and from situation $\mathbf{b}$ to $\mathbf{c}$ as soon as $\varepsilon_{b}$ $>\varepsilon_{c}$, neglecting magnetic hysteresis. After negative saturation of the magnetic soft layer, domain walls of the hard magnetic layer are duplicated in the soft one if $\varepsilon_{b}(H)$ $-\varepsilon_{a}(H)<0$, i.e.,

$$
H>\frac{\sigma_{2} \lambda}{2 M_{s 2} \Delta}-\frac{J_{F}}{M_{s 2} t_{2}}=H_{c r 1} .
$$

This relation can be rewritten as

$$
H_{c r 1}=\frac{h_{\text {wall }}}{\Delta}-H_{d},
$$

where

$$
h_{\text {wall }}=\frac{\sigma_{2} \lambda}{2 M_{s 2}} \text { and } H_{d}=\frac{J_{F}}{M_{s 2} t_{2}} \text {. }
$$

Then, when the positive applied field is increased, the domain structure duplicated in the magnetic soft layer disappears when $\varepsilon_{c}(H)-\varepsilon_{b}(H)<0$, i.e.,

$$
H>\frac{J_{F}}{M_{s 2} t_{2}}-\frac{\sigma_{2} \lambda}{2 M_{s 2}(S-\Delta)}=H_{c r 2} .
$$

This relation can be rewritten as

$$
H_{c r 2}=H_{d}-\frac{h_{\text {wall }}}{S-\Delta} .
$$

Finally, the magnetic state with a duplicated domain structure in layer 2 exists if $H_{c r 2}>H_{c r 1}$, i.e.,

$$
J_{F}>\frac{\sigma_{2} \lambda t_{2}}{4}\left[\frac{1}{\Delta}+\frac{1}{S-\Delta}\right] .
$$

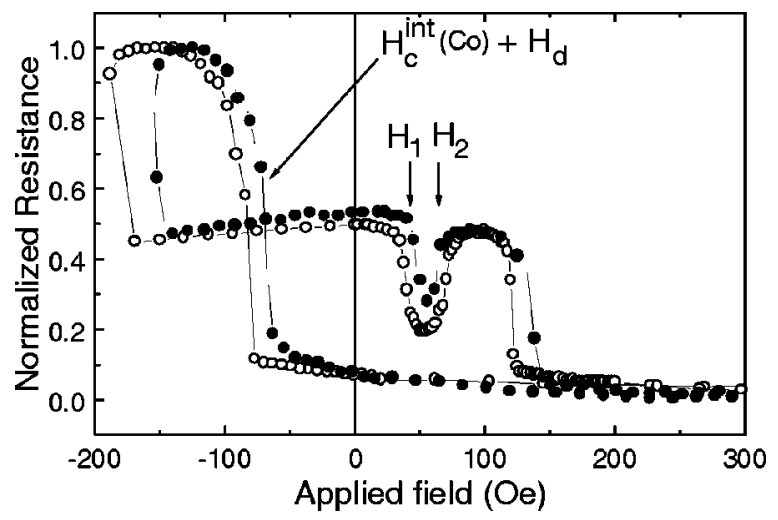

FIG. 3. Two normalized minor tunnel magnetoresistance loops measured on an $\operatorname{AlOx}(1.8 \mathrm{~nm}$, oxidation time $45 \mathrm{~s})\left(-\mathrm{O}_{-}\right)$and an $\mathrm{AlOx}(1.5 \mathrm{~nm}$, oxidation time $35 \mathrm{~s})\left(-0_{-}\right)$tunnel junction made using ex situ changed masks with $200 \mu \mathrm{m}$ lateral size. The two minor cycles have been measured for two $H_{\text {rev }}$ values such that on the $\left(H_{\text {rev }}, 0\right)$ branch, the TMR signal is equal to $\mathrm{TMR}_{\max } / 2$. Since the magnetic properties of the electrodes of the two junctions are the same and due to the shift of $H_{c}^{\text {int }}(\mathrm{Co})+H_{d}$, the electrodes are more strongly coupled when using an $\operatorname{AlOx}(1.8 \mathrm{~nm}$, oxidation time $45 \mathrm{~s})$ tunnel barrier (-O-). The decrease of $H_{d}$ leads to a decrease of $H_{2}-H_{1}$, and so a minimum value of $H_{d}$ is required for duplication to occur.

\section{DISCUSSION}

Equation (8) is of particular importance because it shows that even if a domain structure exists in the hard layer, and so $\Delta$ is different from 0 or $\mathrm{S}$, the duplication occurs only if $J_{F}$ is strong enough. For a given $J_{F}$, Eq. (8) is fulfilled only in a small field window of $H_{\text {rev }}$ as exemplified in Fig. 1. Indeed, on the minor cycle (-O-), no dip between $H_{1}$ and $H_{2}$ can be clearly seen. However, when $H_{\text {rev }}$ is slightly decreased, a dip takes place as can be observed on the minor cycle (-) So, for a given domain structure, $J_{F}$ should exceed a minimum value to allow the duplication.

We are able to reduce the value of $J_{F}$ experimentally by decreasing the "orange peel" coupling between the electrodes. For this, we have decreased the thickness of the alumina barrier. This leads to a smoother tunnel barrier, which in turn reduces the dipolar coupling field between the electrodes down to $12 \mathrm{Oe}$. At the same time, the intrinsic magnetic properties of the electrodes were kept constant. The two minor cycles of Fig. 3 were measured for $\mathrm{AlOx}(1.8 \mathrm{~nm}$, oxidation time $45 \mathrm{~s})\left(-\mathrm{O}_{-}\right)$and $\mathrm{AlOx}(1.5 \mathrm{~nm}$, oxidation time 35 s)(--) tunnel junctions such that on the $\left(H_{\mathrm{rev}}, 0\right)$ branch, the TMR signal is equal to $\mathrm{TMR}_{\max } / 2$. Then, the domain structure in the hard layer is similar in the two measurements when duplication occurs, and so both $\Delta$ and $h_{\text {wall }}$ can be considered constant from one measurement to the other. As expected from Eqs. (5) and (7), $H_{2}-H_{1}$ decreases when $H_{d}$ decreases, and so a minimum value of $H_{d}$ is required for duplication to occur. This value has been estimated to be 6 Oe.

It was stressed in the previous section that this model does not take into account the nucleation process responsible for the reversal of the soft Co layer. Therefore, Eq. (8) is a condition which has to be fulfilled so that duplication would occur, but it is not always sufficient due to the hysteresis effect. Equations (5) and (7) should be modified to include nucleation fields which can be different in each equation. 


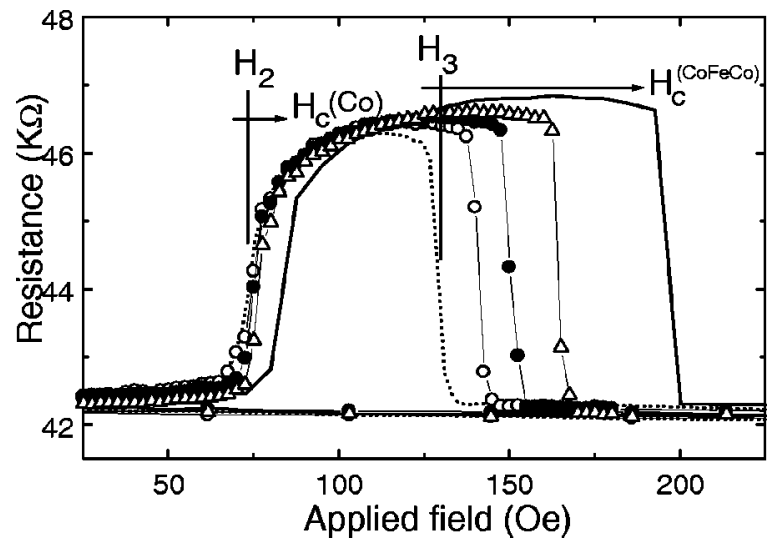

FIG. 4. Positive branch of minor tunnel magnetoresistance loops measured on a $\mathrm{Co}(10 \mathrm{~nm}) / \mathrm{AlOx}(1.8 \mathrm{~nm}$, oxidation time $45 \mathrm{~s}) / \mathrm{Co}(5 \mathrm{~nm}) / \mathrm{Fe}(20 \mathrm{~nm}) /$ $\mathrm{Co}(5 \mathrm{~nm}) / \mathrm{Al}(10 \mathrm{~nm})$ tunnel junction made using ex situ changed masks with $200 \mu \mathrm{m}$ lateral size. The different minor cycles have been obtained with different $H_{\text {rev }}$ with values below $-H_{c}(\mathrm{CoFeCo})$. Decreasing the value of $H_{\text {rev }}$ from -200 Oe (dotted line) to -260 Oe $\left(-\bigcirc_{-}\right),-320$ Oe (--), -430 Oe $\left(-\triangle-\right.$ ), and -850 (continuous line) leads to a smooth drift of $H_{2}$ to $H_{c}(\mathrm{Co})$ and of $H_{3}$ to $H_{c}(\mathrm{CoFeCo})$.

Indeed, from situation $\mathbf{a}$ to $\mathbf{b}$, domains are nucleated in the soft layer and from situation $\mathbf{b}$ to $\mathbf{c}$, domains are nucleated and/or existing domains are propagated in the soft layer. Then, Eqs. (5) and (7) become

$$
\begin{aligned}
& H_{c r 1}=H_{c}^{\mathrm{int}}(\mathrm{Co})-H_{d}+\frac{h_{\mathrm{wall}}}{\Delta}, \\
& H_{c r 2}=H_{p}^{\mathrm{int}}(\mathrm{Co})+H_{d}-\frac{h_{\mathrm{wall}}}{S-\Delta},
\end{aligned}
$$

where $H_{c}^{\mathrm{int}}(\mathrm{Co})$ is the intrinsic coercive field of the soft Co layer and $H_{p}^{\mathrm{int}}(\mathrm{Co})$ is either $H_{c}^{\mathrm{int}}(\mathrm{Co})$ or the intrinsic propagation field of the soft Co layer.

As can be seen in Fig. 1, while $H_{1}$ has always values around $H_{c}^{\text {int }}(\mathrm{Co})-H_{d}, H_{2}$ presents values less than $H_{c}^{\mathrm{int}}(\mathrm{Co})+H_{d}$. This suggests that $H_{p}^{\mathrm{int}}(\mathrm{Co})$ is closer to a propagation field whose value is less than $H_{c}^{\text {int }}(\mathrm{Co})$ in our samples. The fact that no new domain is nucleated at $\mathrm{H}_{2}$ and that propagation of existing domains is the outstanding reversal process has been shown in an earlier study. ${ }^{6}$ However, a significant difference between $H_{2}$ and $H_{c}(\mathrm{Co}), H_{3}$ and $H_{c}(\mathrm{CoFeCo})$ persists even if around $H_{\mathrm{rev}}$ in Fig. $1(-\diamond-)$, the resistance of the junction is minimal, and so the magnetizations of the electrodes in the junction area are saturated and parallel. To shed light on this discrepancy, we have studied the evolution of $\mathrm{H}_{2}$ and $\mathrm{H}_{3}$ when $\mathrm{H}_{\text {rev }}$ was decreased from $-H_{c}(\mathrm{CoFeCo})$ down to $-H_{\text {sat }}$, the saturating field used in this study equaled to $-850 \mathrm{Oe}$. When $H_{\text {rev }}$ is in this field window, the resistance of the junction on the $\left(H_{\text {rev }}, 0\right)$ branch is minimum. We can see in Fig. 4 that as $H_{\text {rev }}$ decreases, $\mathrm{H}_{2}$ and $\mathrm{H}_{3}$ gradually increase towards $H_{c}(\mathrm{Co})$ and $H_{c}(\mathrm{CoFeCo})$, respectively. At the same time, the amplitude of the resistance variation increases and reaches its maximum when $H_{\text {rev }}=-H_{\text {sat }}$.

The increase of $H_{3}$ towards $H_{c}(\mathrm{CoFeCo})$ in the case of junctions with cross geometry is related to residual domains

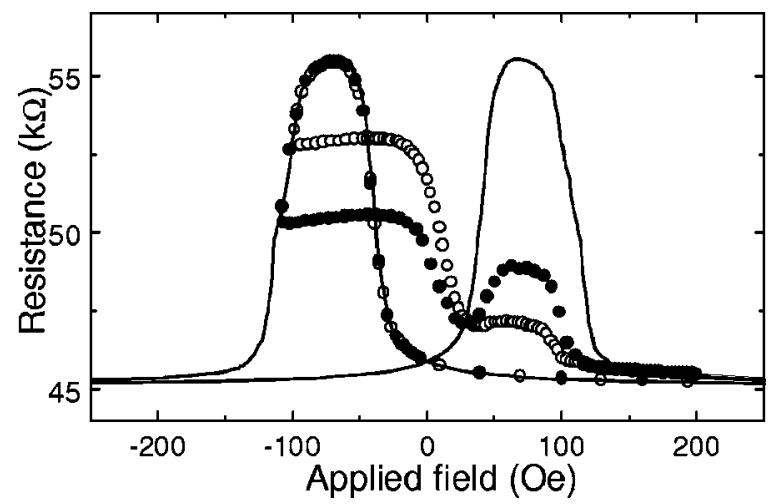

FIG. 5. Complete ( - ) and two minor tunnel magnetoresistance loops measured on a $\mathrm{Co}(10 \mathrm{~nm}) / \mathrm{AlOx}(2.1 \mathrm{~nm}$, oxidation time $45 \mathrm{~s}) / \mathrm{Co}(5 \mathrm{~nm}) / \mathrm{Fe}(20$ $\mathrm{nm}) / \mathrm{Co}(5 \mathrm{~nm}) / \mathrm{Al}(10 \mathrm{~nm})$ tunnel junction made using lithography. The junction is a disk with a diameter of $50 \mu \mathrm{m}$. Here, we can see that $H_{2}$ is always around $H_{c}(\mathrm{Co})$ and $H_{3}$ is always around $H_{c}(\mathrm{CoFeCo})$. Note the increase of the TMR signal from $10 \%$ to $20 \%$ when the interfaces of the junction are not exposed to the air during the change of the ex situ shadow masks.

or misaligned magnetic moments that are stored along the magnetic hard electrode outside the junction area. Those magnetic defects are blocked due to the shape of the electrodes. Indeed, since a gap between the shadow mask and the sample during film deposition always exists, the cross section of the electrodes is not rectangular. Instead, they have rounded edges due to deposition of material under the mask by atoms incoming nonperpendicularly to the film plane. The thinnest parts of the electrode are more difficult to saturate, and they constitute favorite nucleation centers during the hard electrode reversal. Their saturation leads, then, to an increase of the nucleation field of the hard electrode. Nonsaturation of the CoFeCo electrode can have an impact on the intensity of the dipolar coupling with the soft electrode. Gradual saturation of misaligned magnetic moments leads to an increase of the dipolar interaction with the soft Co layer and, therefore, to a shift of $\mathrm{H}_{2}$ towards $H_{c}(\mathrm{Co})$. Nevertheless, the shift of $H_{2}$ towards $H_{c}(\mathrm{Co})$ can also be explained by a gradual saturation of the soft electrode magnetization since it is also made using a shadow mask. The same arguments of nonsaturation invoked for the hard electrode then also can be applied.

One way to highlight the effect of the residual domain structures stored along the electrodes outside the junction area is the use of lithography to make tunnel junctions. In this case, and as discussed in Sec. II, the top electrode is cut during an ion-milling step to allow an electrical contact to the bottom electrode. Then, the top hard and bottom electrodes are confined to a disk and a dumbbell-shaped electrode, respectively, with a straight profile. So, in those samples the magnetic response is better controlled: (i) the edges of the patterns are less rounded and residual domains are easier saturated, and (ii) the junction resistance fully reflects the magnetism of the hard electrode since its area is restricted to that of the junction; there is no way to store residual domains in the hard electrode outside the junction area. As can be seen in Fig. 5, $H_{1}$ has values always around $H_{c}^{\text {int }}(\mathrm{Co})-H_{d}$ and $H_{2}$ always presents values around $H_{c}^{\text {int }}(\mathrm{Co})+H_{d}$. This means clearly that the trends of $H_{2}$ and 
$H_{3}$ depicted on the junctions made with the ex situ changed masks are related to residual domain structures stored in the hard electrode. Nevertheless, duplication of the domain structure is confirmed, as exemplified in Fig. 5. Finally, it appears that in our junctions $H_{c}^{\text {int }}(\mathrm{Co})$ and $H_{p}^{\text {int }}(\mathrm{Co})$ have similar values and the model gives a satisfactory description of the experimental results.

\section{CONCLUSIONS}

In this article, we give an overview of the domain duplication process which can occur in ferromagnetic sandwiches. A brief theoretical description of the process allows us to extract the main parameters governing the effect. It is shown that a minimum ferromagnetic coupling $J_{F}$ is required and that even if a domain structure exists in the hard electrode, no duplication can occur if $J_{F}$ is too small. Finally, the effects of residual domains in the hard electrode have been addressed to reconcile theory and experiment.

\section{ACKNOWLEDGMENTS}

The authors thank M. Alnot, J. Latriche, and P. Schmitt for their technical support. The authors thank F. Nguyen van Dau for many fruitful discussions and P. Rottländer for careful reading of the manuscript.

${ }^{1}$ L. Thomas, J. Lüning, A. Scholl, F. Nolting, S. Anders, J. Stöhr, and S. S. P. Parkin, Phys. Rev. Lett. 84, 3462 (2000).

${ }^{2}$ C. Tiusan, T. Dimopoulos, M. Hehn, V. Da Costa, Y. Henry, H. A. M. van den Berg, and K. Ounadjela, Phys. Rev. B 61, 580 (2000).

${ }^{3}$ L. Néel, C. R. Acad. Sci. URSS 255, 1676 (1962).

${ }^{4}$ C. Cowache, B. Dieny, S. Auffret, M. Cartier, R. H. Taylor, R. O’Barr, and S. Y. Yamamoto, IEEE Trans. Magn. 34, 843 (1998).

${ }^{5}$ M. Hehn, O. Lenoble, D. Lacour, C. Féry, M. Piécuch, C. Tiusan, and K. Ounadjela, Phys. Rev. B 61, 11643 (2000).

${ }^{6}$ O. Lenoble, M. Hehn, D. Lacour, A. Schuhl, D. Hrabovsky, J. F. Bobo, B. Diouf, and A. R. Fert, Phys. Rev. B 63, 052409 (2001).

${ }^{7}$ M. Hehn, O. Lenoble, D. Lacour, and A. Schuhl, Phys. Rev. B 62, 11344 (2000).

${ }^{8}$ F. Montaigne, J. Nassar, A. Vaurès, F. Nguyen Van Dau, F. Petroff, A. Schuhl, and A. Fert, Appl. Phys. Lett. 73, 2829 (1998). 